\title{
A note about reproducibility in visual ITC
}

\author{
L. Garrigue ${ }^{1 *}$ and Laurence Lecot $^{\dagger}$ \\ ${ }^{1}$ École des ponts ParisTech, France \\ ${ }^{1}$ Institut français de recherche et d'expérimentation spirite (Ifres), Paris, France
}

(Dated: September $28^{\text {th }}, 2021$ )

\begin{abstract}
We present several orders of magnitude of some anomaly occurring rates $f$ that we obtained in visual instrumental transcommunication. We used two techniques, the first one is numeric and relies on Perlin noise, it was run by the second author who presents solid mediumship and obtained $f \sim 10^{-3}$. The second one is a reproduction of a mist technique developed by the Institut français de recherche et d'expérimentation spirite (Ifres), it was run by the first author who has no mediumistic ability and obtained $f \sim 10^{-5}$.
\end{abstract}

\section{INTRODUCTION}

Instrumental transcommunication (ITC) denotes the attempts to record "intelligently created" anomalies by using modern instruments and, most often, randomly fluctuating physical systems. Let us denote by Supposedly Immaterial Entity (SIE) any source of force, light, or information which cannot be explained by current understanding of physics, and which is usually responsible for the so-called paranormal phenomena, including ITC. Those SIEs could be "spirits", they could be materialized thoughts created by our own psyche, or have other interpretations, and we do not know how they act on physical systems.

In 1920, Thomas Edison reported that he wanted to provide some adapted apparatuses to psychics, to help them in their researches on afterlife communication [15]. In the 1950's, Attila von Szalay and then Friedrich Jürgenson claimed to have recorded voices from an unknown source. Since then, many have received such audio communications $[4,13,20,23]$. Another communication channel is available through videos and images, this is commonly called visual ITC. To the best of our knowledge, the first "anomalous" images were recorded in 1985 by Klaus Schreiber [4, 23] using a television set broadcasting the output of a camera filming the television, forming a feedback loop [23]. More resources on ITC results can be found in $[1,2,4,6-9,11,14,21,24]$ for instance, and resources on mediumship can be found in $[3,5,10,12,16,17,22]$.

Widespread visual ITC techniques use televisions, liquid-air interfaces, glass and other reflective materials, or vapor, for instance. It is relatively easy to learn visual ITC techniques from dedicated social network groups or existing literature $[11,21,23,25]$. Nevertheless, from those sources of information, it is not always easy to extract an idea of the possible anomalies probability of occurrence when starting the experiments. This note aims at providing instances of orders of magnitude of those rates, that we obtained in two different settings, and also discuss qualitative aspects.

\footnotetext{
*https://cermics.enpc.fr/ garrigul

$\dagger$ lecot.laurence@laposte.net
}

We used numerical Perlin noise, run by the second author who is medium, and we reproduced Ifres' mist experiment, run by the first author who is not medium. We respectively obtained occurring rates of anomaly of about one image in $10^{3}$ in the first experiment, and one in $10^{5}$ in the second one.

\section{A REPRODUCTION OF A PERLIN NOISE DEVICE RUN BY A MEDIUM}

\section{A. The software used}

In [19], Ken Perlin developed an algorithm aiming at reproducing natural textures such as clouds or coastal topologies from randomness. To the best of our knowledge, the first software dedicated to visual ITC used Perlin noise and was implemented by Martin Bellefeuille, from ideas of some of his collaborators, and he encapsulated it into an application called Spektrocom. Since then, several applications using artificially created noise have been made available. Strong results using this technique by several experimenters showed that visual ITC can be practiced without any physical apparatus, where everything is generated from short algorithms and pseudo-randomness.

We used Javascript and HTML in all our codes so that experimenters only need a browser, hence facilitating collaborations. All the operations for generating the Perlin noise are done in the experimenter's computer. The core or the Perlin code was classical, written in Javascript and made publicly available by Denis Sibilev. We applied it to produce Perlin noise monolayers, we superposed several layers corresponding to different colors and spatial frequencies (called harmonics), and we made it available on the website itc-station.org. The device constantly generates new pictures, which are completely independent from each other since this is a purely spatial 2-dimensional Perlin noise, not 2-dimensional in space and 1-dimensional in time progressively evolving. The resolution of the generated images is proportional to the resolution of the experimenter's computer resolution, and in our experiment, it typically consisted of $1 \cdot 10^{3} \times 5 \cdot 10^{2}$ pixels. The time to generate one picture typically lies between 2 and 15 seconds, depending on the computational power and on the parameters that were chosen. 
We established an interface enabling the user to change many parameters: contrast, luminosity, zoom, numbers of harmonics, colors, for each layer. A large number of degrees of freedom selectable by the user enables the creation of very diverse textures. We made available several combinations of predefined settings, buttons to switch to full-screen, to download, and to automatically download pictures each time a new one is generated. In predefined parameters, the luminosity of the different layers decreases exponentially with the harmonics, forming fractal-like structures. The last few generated pictures are stored in the webpage in case the experimenter lacks time to analyze or download one of them. The interface design enables each experimenter to choose and store the configurations, and tries to ease as much as possible the use of the device. The source code of the software is also public.

Our source of randomness is Javascript's Math.random() function, which returns a pseudorandom number uniformly distributed between 0 and 1 , generated by the experimenter's computer. Generally, browsers interpret this function by first looking at the system clock to obtain an initial number and then process it through the xorshift128+ algorithm.

\section{B. About the mediumship of the experimenter}

The second author has been practicing ITC since 2007, mainly using audio channels. She never really practiced visual ITC prior to this experiment. She has had a neardeath experiment and saw many times partially or fully materialized SIEs, and from her personal experience, she is convinced that those entities are external spirits living in "other physical spaces". She also experienced rare paranormal and mediumistic events, showing that she has strong mediumistic abilities. We remark that her presence strengthens ITC anomalies production.

\section{Practices during a session}

During sessions, the second author listens to music. In the first part of a session, she reaches a state of mind in which she feels to be in contact with SIEs. To reach this receptive state, she meditates, prays, sings, until being relaxed, and drown with emotions like joy and feelings of "connection" with something "universal". She then sees visual "mirages", or shades, as if some small regions of the surrounding air of the room had variations in their light index. The "mirages" or shades she sees measure several centimeters, they move very fast, rarely stay still, and other present people do not see them. She feels that they are spirits engaging her.

During those visual ITC sessions, she feels in communion with them. She feels warmth in her body, particularly in her fingers, and sweats. She feels that there is a bond and an exchange of positive emotions between them, as if they knew her and she knew them. Further-

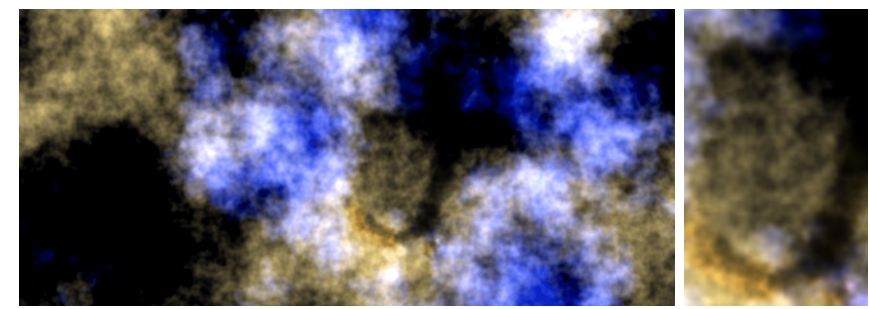

FIG. 1. A complete Perlin picture and its zoom on the anomaly.

more, she feels that they are also linked with each other, but not the same way as with her. In case there is negativity, or even aggressiveness in some of them, she cannot and does not want to establish a link, and hence stops the interaction. She also feels some others in need but without aggressiveness, in which case she tries to appease them.

In this state of mind, she also has mediumistic visions of faces. With the Perlin noise device, she feels those connections more easily than with audio ITC streams, even if she is more used to audio ITC. This is probably due to the fact that the visual stream is more concrete. Moreover, when she practices ITC with video and audio at the same time, the feeling of the "connection" with SIEs is even faster, everything seems fluid to her, like in a real conversation. When a vision of a face occurs in her mind, she almost immediately has a strong emotion. Information given by such visions of some faces had already allegedly been confirmed by photos, which information could not have been previously known by her, providing her evidence for clairvoyance abilities and consolidating her in the belief of her own mediumship. She also thinks that she witnessed correlations between her clairvoyance visions and her visual ITC results.

At the end of a session, she is either very tired, or feels an overflowing strength enabling her not to sleep for a long period.

\section{Results}

We assembled our device in January 2021, and the results we present here have been recorded since then. In this section, we present the most significant anomalies that the second author observed. Over this period of approximately 8 months, she made 47 sessions of typically one hour, and analyzed about $1.4 \times 10^{4}$ pictures overall. In the images we present in this section, we did not change the luminosity or the constrast.

In Figures 1 and 2, we present an example of a wholly generated image and its zoom on the supposed anomalies. In Figure 3, we present other faces obtained, zoomed on the anomalies. 

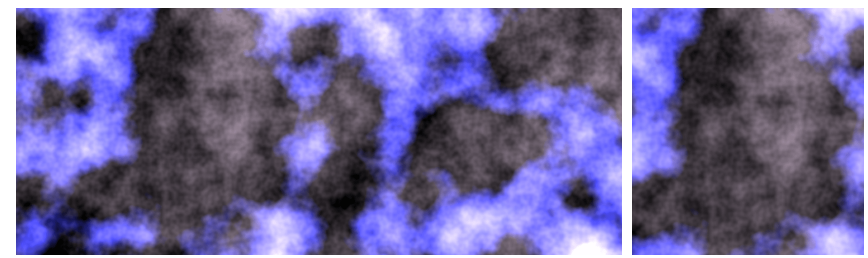

FIG. 2. Another complete Perlin picture and its zoom on the anomaly.
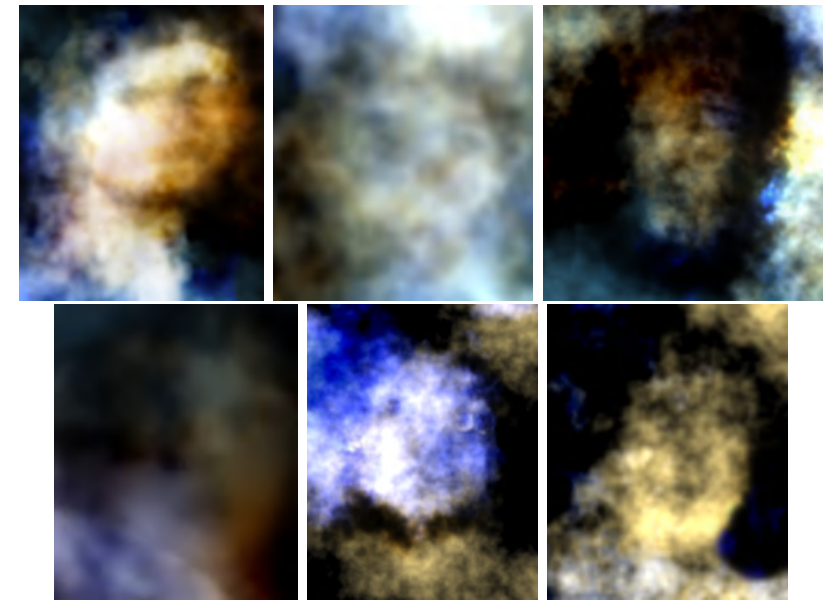

FIG. 3. Anomalies recorded with Perlin noise.

The other kind of anomalies is the presence of letters and numbers, similarly as in Ifres' results. We present instances on Figures 4, 5, and 6. On the left picture of Figure 4, we feel to be able to read the three letters "Ame", meaning "Soul" in French (the mother tongue of the second author). On the right picture of Figure 4, we think to see the two letters "TC" in upper case. On Figure 6, we think to see on the left three letters forming "ITC", and this is the strongest letter anomaly she obtained. Most of the time, the letters seem to be created by a thin white line.

Retaining only the 12 previous images in the $1.4 \times 10^{4}$ generated and observed, we can deduce a rate of appearance of such anomalies in this set of experiments to be of the order of magnitude of one image in $10^{3}$.
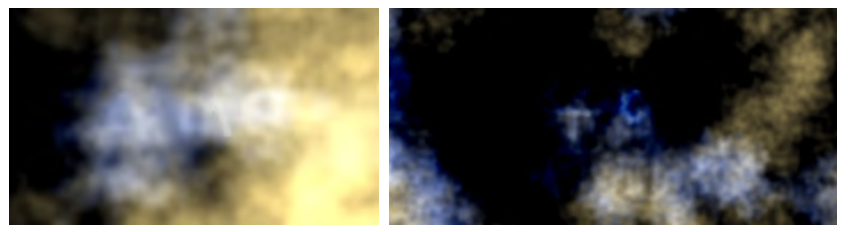

FIG. 4. Letters anomalies in Perlin noise.

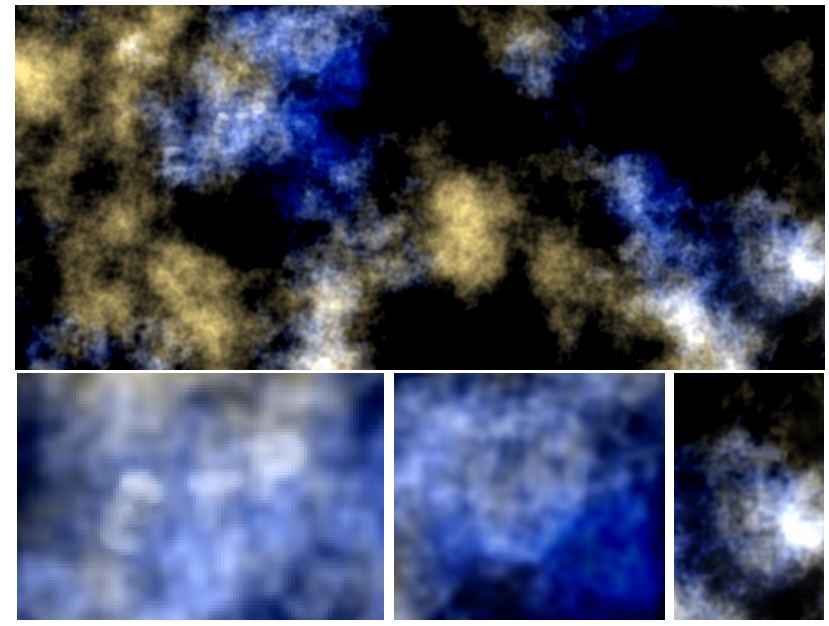

FIG. 5. Simultaneous letters and face anomalies in Perlin noise.

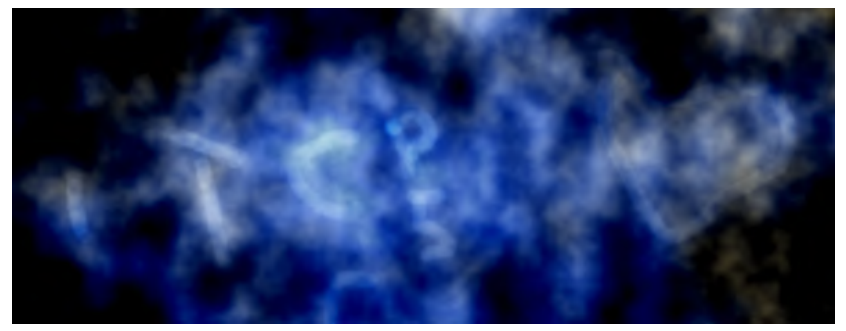

FIG. 6. Strongest letter anomaly obtained in Perlin noise.

In comparison, the first author also produced and analyzed Perlin pictures, $5.0 \times 10^{3}$ during experiments in which he was present and observing, and $1.5 \times 10^{4}$ which were generated without his presence but analyzed a posteriori. He never witnessed any anomaly of the same scale as in the session of the second author.

\section{A REPRODUCTION OF IFRES' TECHNIQUE RUN BY A NON-MEDIUM EXPERIMENTER}

\section{A. Ifres' setting and some examples}

The association Ifres has been practicing visual ITC for the last 30 years and one of the founders, who has been present at all sessions, presents solid mediumistic abilities. Since 1998, hundreds of recorded anomalies have been displayed on the ifres.org webpage, the setups were also made public since then. In Figure 7, and to compare them to our results, we present some recordings obtained by the association.

The heart of Ifres' technique is the use of artificial mist, created by a classical mist maker, filmed and lighted by a green laser and a videoprojector. Strioscopy is also used, which increases the duration of visibility of the anomalies, as well as other assemblies. Formerly, the projector displayed the camera stream, realizing a Klaus Schreiber 

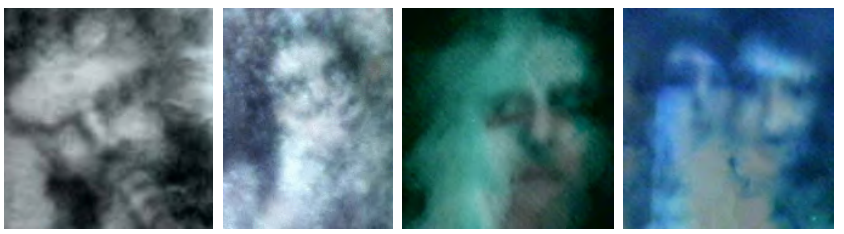

FIG. 7. A few recordings from Ifres.

feedback loop, but since more recently, it projects small white dots randomly positioned. The filmed area is a square of physical size $15 \mathrm{~cm} \times 15 \mathrm{~cm}$, and the recording rate is 80 frames per second. The sensor of the camera is a Sony IMX 290, particularly efficient in low light conditions compared to sensors of the same range.

During the sessions, video sequences of 10 seconds are filmed and then analyzed frame by frame. Usually, 5 such sequences are recorded during each session. The rate of anomalies is about one in $10^{2}$ images, and those anomalies are qualitatively very apparent. Each appearance lasts between 20 and $40 \mathrm{~ms}$, otherwise the mist is very homogeneous and no structure can be recognized. The anomalies are symbols, numbers, letters, animal faces, but mostly human faces. The main experiment is located near Paris, and a reproduction of that experiment, made by Ifres and located at Dijon, obtains results of similar robustness. It is still an open question to understand if anomalies are produced by a change in the geometry of the mist and/or in a transformation of incident light.

\section{B. Our reproduction}

The first author, who has no particular mediumistic ability, reproduced a subset of Ifres' experiment, corresponding to the setup presented in Section III A, and ran it in his laboratory, independently from Ifres. We only used a green laser and neutral videoprojector lights projected on an artificial mist, and we used the same sensor as Ifres. Recordings were taken from 2018 to 2020. As in the original experiment, the texture of the artificial mist is very homogeneous. The filmed area was placed in a box, and the experiments were mostly conducted in the absence of light other than the one produced by the instruments. The only two operations applied to the original recordings, compared to the presented images, are cropping and uniform modifications of luminosity. Finally, the first author was almost always alone during the sessions.

During this investigation, the number of recorded images was overall about $7 \times 10^{5}$. Figures 8, 9, 10 and 11 show the 8 most relevant anomalies, to our opinion. The order of magnitude of the rate of anomaly apparitions in this experiment can hence be estimated to be one in $10^{5}$.

We think that the most significant anomaly that was received is the one presented in Figure 8, on which we can distinguish a well-proportioned facial profile, with eyes, eyes contours, eyebrows, a nose, a line forming the lips, a chin, and hair.

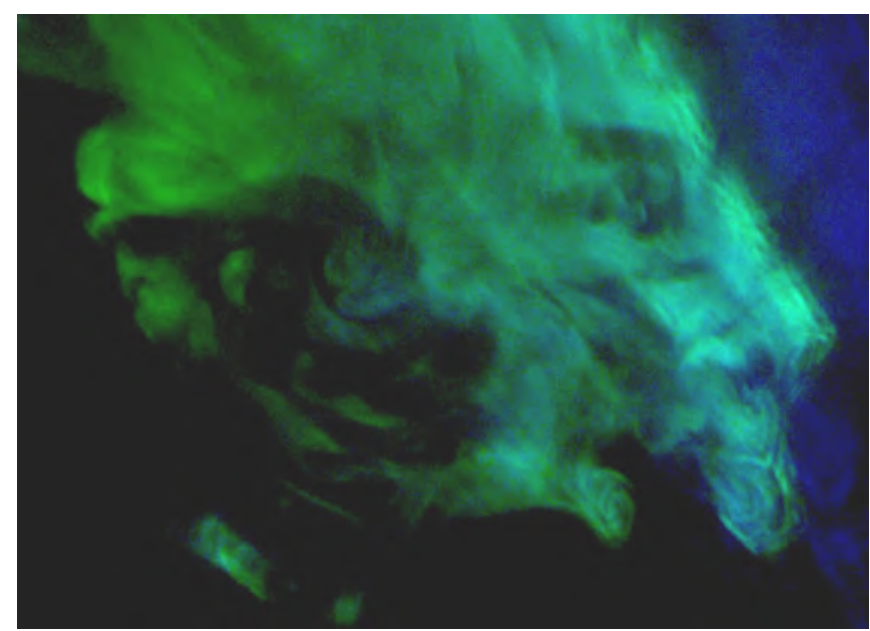

FIG. 8. Most precise recording received by the first author

In those recordings, when mouth and nose cannot be recognized, we most often detect an anomaly because eyes are precisely formed, sometimes accompanied by eyebrows, as illustrated in Figures 9, 10 and 11. More generally, the eyes are also the part of the face that is the most detailed. We saw many slightly weaker anomalies presenting an eye.

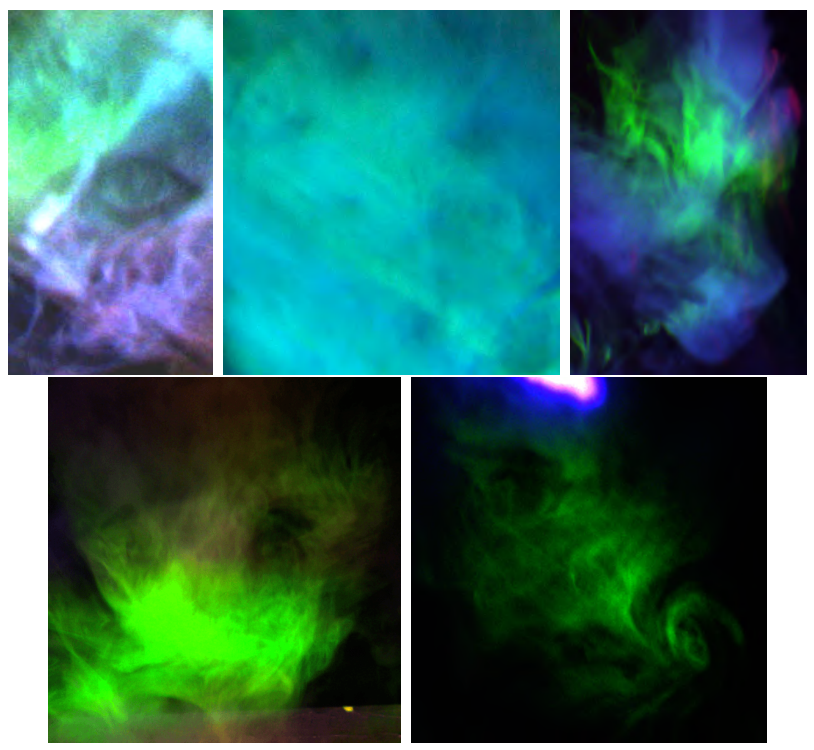

FIG. 9. Anomalies mainly formed by the presence of eyes. 

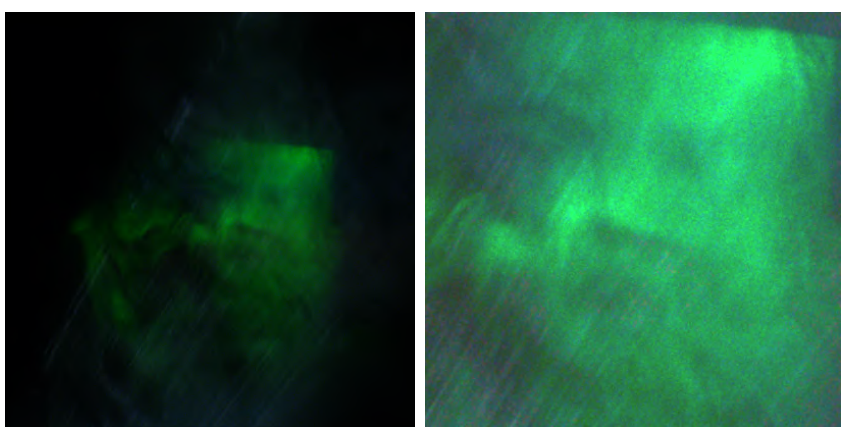

FIG. 10. Global image of a face anomaly, and its zoom on the face.
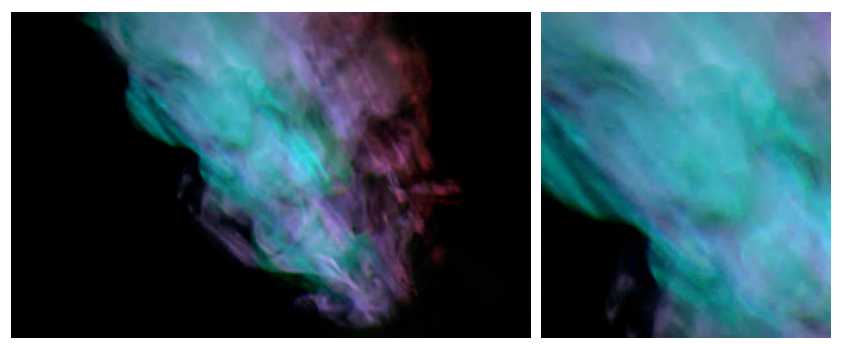

FIG. 11. Another image of a face anomaly, and its zoom on the face.

We tried to apply a standard machine learning software on the pictures to automatize face detection, but this did not significantly help since the anomalies are not strong enough. Hence, we analyzed the images by directly observing all of them. With more expertise in visual detection, it might be possible to identify the anomalies via the presence of eyes.

\section{Comparision to Ifres' results}

Ifres' results are much more significant, both quantitatively and qualitatively. Indeed, during the sessions of the association, there is one strong anomaly every 3 seconds of recording, approximately, giving roughly a rate of appearance of one anomaly every $2 \times 10^{2}$ images. Ifres' anomalies rate is hence about $10^{3}$ times higher, and the anomalies are much clearer than in our case, objectively showing the existence of those phenomena.

The participation of the first author to Ifres' mediumistic and ITC sessions might also have contributed to improve the results of this presented study.

\section{DISCUSSION}

\section{A. Pareidolia}

We recall that some of the images presented in this document could have been misinterpreted as being anomalies by the authors, constituting so-called pareidolia. Moreover, zooming on the recognizable parts can also trigger pareidolia. Nevertheless, considering the degree of homogeneity of the mediums used (mist and Perlin noise) the rate of apparition of the faces and letters, and the fact that other forms do not appear, we think that unknown physical actions contributed to create them.

\section{B. Forms of the anomalies in mist and Perlin}

First, we remark that the mist setup is much more homogeneous than the Perlin noise device. Moreover, in the mist experiment, all the anomalies we obtained are faces, contrarily to Ifres' case, in which symbols, animals, numbers and letters appear.

In the mist and Perlin noise experiments, we recognize curved structures as supports of the faces, then darker regions form eyes, the contours of the nose, sometimes eyebrows, and hair. Usually, the most specific part of the face is one or two eyes. Often, faces are in the frontal position or slightly turned, and Figure 8, where the face is in the profile position, is an exception.

By symmetry, and even taking into account that the human brain is particularly trained to recognize faces, we should see all other structures and objects if the anomalies were explainable by pareidolia. But the only recognizable ones are faces and letters, and we never witnessed any other forms. This is one of our main arguments to support that these supposed anomalies are not explainable by current physics.

\section{Reproducibility}

In previous sections, we saw an instance of a nonmedium person seemingly managing to obtain anomalies, but which are several orders of magnitude rarer than at Ifres. We also saw an instance of a medium receiving visual anomalies with the Perlin noise while a non-medium does not. Reproducibility is the greatest issue in ITC. One can witness strong results in colleagues experiments, for instance in strong "bridges" like at Ifres, the anomalies are so apparent that they are detectable by classical face recognition softwares. But one will still seemingly need extensive efforts to try to replicate them.

The challenge at stake is to understand the right conditions improving the "receiving channel", in terms of both image quality and quantity. From Ifres' 30 years experience, it seems that establishing a strong channel is made by a combination of teamwork, regularity, mediumship and persistence. The conditions of establishment of a strong "bridge" with SIEs are discussed in the books of Foy $[8,9]$.

It is also important to remark that provoking paranomal phenomena is possible without any mediumistic element, one interesting instance is the experiment described in [18]. This also seems to be the case in our reproduction of Ifres' experiment.

Another element which seems to improve an ITC "connection" are strong emotional bonds. Bereaved people 
wanting to receive messages from their relatives seem to obtain such connections more easily. Moreover, people posting their results on social networks seem to detect more anomalies, a reason could be that the phenomenon wants to manifest to one of the contacts of the experimenter, who will see the published result, so that the third person is informed of the anomaly, which might be a "message" in such cases. Yet we note that at Ifres, this emotional bond does not seem to be necessary.

Finally, the production of anomalies probably also highly depends on the setup and on the quality of the devices. Some sources of randomness could be more "manipulable" than others by SIEs. The physical systems used need to naturally generate fluctuations which can possibly, at a relevant rate, produce faces, letters, numbers. This natural rate is the main trade-off that we can control, and we expect the SIEs to significantly increase it by their unknown action. If the natural rate is too high, pareidolia dominates and it becomes hard to distinguish between paranormal action and natural fluctuations, but if the natural rate is too low, then the physical system might not be malleable enough for the SIEs.

\section{An ethical issue}

Even in a weak "bridge", good software and hardware could enable to detect such anomalies automatically. But this raises ethical questions: what if millions of experimenters try to obtain anomalous images for entertainment for instance? While ITC seems safe for us, could such actions perturb SIEs in case they are external entities?

\section{ACKNOWLEDGEMENT}

We thank Ifres for sharing their techniques, and for permitting the presentation of some of their images in this document. The first author is deeply indebted to Ifres for having shown him the reality of ITC. We are also grateful to Keith J. Clark for having informed us that visual ITC can be practiced by using Perlin noise, Karyn Jarvie for having helped us with language corrections, and Michael S. Lee for useful comments. Finally, we thank Denis Sibilev for having made public his Perlin noise implementation.

Support from the Society for Psychical Research, the Helen Reeder fund, and from Valérie Seguin is acknowledged.

The names of the authors are presented in alphabetical order, and the first name of the first author is not indicated for academic protection reasons.
[1] M. BACCI, Il mistero delle voci dell'aldilà, Edizioni Mediterranee, 1985.

[2] I. BARUŠs, An experimental test of instrumental transcommunication, J. Sci. Explor, 21 (2007), p. 89.

[3] M. A. V. Bastos, P. R. H. D. O. Bastos, L. M. Gonçalves, I. H. S. Osório, And G. Lucchetti, Mediumship: review of quantitatives studies published in the 21st century, Arch. Clin. Psychiatry (São Paulo), 42 (2015), pp. 129-138.

[4] F. Brune, Les morts nous parlent, Tome 1, Le Livre de Poche, 2009.

[5] E. Cardeña, J. PAlmer, And D. MarcussonClavertz, Parapsychology: A handbook for the 21st century, McFarland, 2015.

[6] A. CARdoso, Electronic voices: contact with another dimension?, John Hunt Publishing, 2010.

[7] S. W. Estep, Voices of eternity, Fawcett Gold Medal, 1988.

[8] R. Foy, In Pursuit of Physical Mediumship, Janus Publishing Company Lim, 2007.

[9] — Witnessing the impossible, Torcal publications, 2008.

[10] A. Gauld, Mediumship and survival: A century of investigations, David and Charles, 2012.

[11] J.-M. GRANDSIRE, La transcommunication, JMG éditions, 1998.

[12] A. Kardec, Le livre des médiums, Didier \& Cie, 1861.

[13] H.-O. KÖNIG AND A. M. Wauters, Listen! New discoveries about the afterlife, CreateSpace edited by Craig Hogan, 2017.
[14] E. LAszLO, An unexplored domain of nonlocality: toward a scientific explanation of instrumental transcommunication, Explore, 4 (2008), pp. 321-327.

[15] A. C. Lescarboura, Edison's views on life and death, Sci. Am, 123 (1920), pp. 446-460.

[16] M. Meilleur, Great Moments of Modern Mediumship, Volume 1, Saturday Night Press, 2014.

[17] — Great Moments of Modern Mediumship, Volume 2, Saturday Night Press, 2021.

[18] I. M. Owen AND M. Sparrow, Conjuring up Philip: An adventure in psychokinesis, HarperCollins Publishers, 1976.

[19] K. Perlin, An image synthesizer, ACM Siggraph Computer Graphics, 19 (1985), pp. 287-296.

[20] K. RAUdIVE, Breakthrough: An amazing experiment in electronic communication with the dead, Smythe Buckinghamshire, UK, 1971.

[21] S. Rinaldi, Contatos interdimensionais, Editora Pensamento, 2005.

[22] A. J. Rock, The survival hypothesis: Essays on mediumship, McFarland, 2014.

[23] H. SCHÄFER, Théorie et pratique de la transcommunication: un pont entre notre monde et l'au-delà, R. Laffont, 1992.

[24] G. E. Schwartz, Photonic measurement of apparent presence of spirit using a computer automated system, Explore, 7 (2011), pp. 100-109.

[25] M. Simonet, Images et messages de l'au-delà, Rocher, 1992. 